\title{
Respiratory disease and climatic seasonality in children under 15 years old in a town in the Brazilian Amazon
}

\author{
Doença respiratória e sazonalidade climática em menores de 15 anos em um \\ município da Amazônia brasileira
Antonia Maria Rosa ${ }^{1}$, Eliane Ignotti ${ }^{2}$, Clóvis Botelho ${ }^{3}$, Hermano Albuquerque de Castro ${ }^{4}$, Sandra de Souza Hacon ${ }^{5}$

\begin{abstract}
Resumo
Objetivo: Analisar a sazonalidade climática dos atendimentos ambulatoriais por doença respiratória (DR) em menores de 15 anos.

Métodos: Estudo epidemiológico descritivo, realizado com dados dos Registros de Ocorrências Ambulatoriais das unidades básicas de saúde referentes ao período 2004-2005, do município de Tangará da Serra (MT). As estimativas populacionais foram obtidas do Instituto Brasileiro de Geografia e Estatística (IBGE), e os dados de umidade relativa do ar e temperatura, do Instituto Nacional de Meteorologia (INMET). Foram construídas taxas médias de atendimento ambulatorial por DR segundo sexo, faixa etária e localização anatômica. Calculou-se a razão seca/chuva dos atendimentos de acordo com a localização anatômica da DR. Utilizou-se o Epi-Info 3.2 para análise dos dados, com as diferenças de proporção testadas através do teste do qui-quadrado, ao nível de significância de 5\%.
\end{abstract}

Resultados: Indivíduos do sexo masculino tiveram taxa quase $50 \%$ maior $(37,3 / 25,0)$ de atendimentos ambulatoriais por doenças das vias aéreas inferiores que os do sexo feminino. As taxas de atendimentos ambulatoriais por DR em menores de 15 anos apresentaram variação com o aumento da idade, passando de $457,7 \%$ o nos menores de 1 ano de idade para $133,5 \%$ o no grupo de 10 a 14 anos. Os atendimentos por DR foram em média $21 \%$ menos freqüentes(4.148/5.231) no período seco $(p=0,000)$. Observou-se picos de atendimento nos meses de março e agosto, mais acentuados em março, período chuvoso na região.

Conclusão: Os atendimentos ambulatoriais por DR, especialmente por doenças das vias aéreas superiores, estão relacionados ao período chuvoso no município.

J Pediatr (Rio J). 2008;84(6):543-549: Doenças respiratórias, sazonalidade climática, doenças das vias aéreas superiores, doenças das vias aéreas inferiores

\begin{abstract}
Objective: To analyze the climatic seasonality of primary care visits for respiratory disease (RD) in children less than 15 years old.

Methods: This was a descriptive, epidemiological study based on data from the municipal records of primary care events from basic healthcare centers for the period 2004-2005, for the municipality of Tangará da Serra (MT), Brazil. Population estimates were obtained from the Brazilian Institute of Geography and Statistics (Instituto Brasileiro de Geografia e Estatística, IGBE), and data on temperature and relative humidity of the air, from the National Meteorology Institute (Instituto Nacional de Meteorologia, INMET). Mean rates of primary care visits for RD were calculated according to sex, age group and anatomic location of complaint. The ratio of dry season to rainy season visits was calculated according to anatomic location of the RD. Data were analyzed using Epi-Info 3.2, testing differences between proportions using the chi-square test to a significance level of $5 \%$.
\end{abstract}

Results: Male children had an almost 50\% greater (37.3/25.0) rate of primary care visits for diseases of the lower respiratory tract than did females. The rates of primary care visits due to RD in children under 15 years of age varied as age increased, varying from $457.7 \%$ of children less than 1 year of age to $133.5 \%$ in the 10 to 14 years-of-age group. During the dry season there were an average of $21 \%(4,148 / 5,231)$ fewer visits for $R D(p=0.000)$. Peaks in numbers of visits were observed during the months of March and August, being more accentuated in March, which is the wet season in the region.

Conclusion: Primary care visits for RD, especially those due to upper airway diseases, are related to the rainy season in this municipality.

J Pediatr (Rio J). 2008;84(6):543-549: Respiratory diseases, climatic seasonality, upper airway diseases, lower respiratory tract diseases climate, season.

1. Mestre. Professora, Universidade do Estado de Mato Grosso (UNEMAT), Cáceres, MT.

2. Doutora. Professora, UNEMAT, Cáceres, MT. Instituto de Saúde Coletiva, Universidade Federal de Mato Grosso (UFMT), Cuiabá, MT.

3. Doutor. Professor, UFMT, Cuiabá, MT.

4. Doutor. Professor, Escola Nacional de Saúde Pública, Fundação Oswaldo Cruz (Fiocruz), Rio de Janeiro, RJ.

5. Doutora. Professora, Escola Nacional de Saúde Pública, Fiocruz, Rio de Janeiro, RJ.

Apoio financeiro: Conselho Nacional de Desenvolvimento Científico e Tecnológico (CNPq) e Fundação de Amparo à Pesquisa do Estado de Mato Grosso (FAPEMAT).

Não foram declarados conflitos de interesse associados à publicação deste artigo.

Como citar este artigo: Rosa AM, Ignotti E, Botelho C, de Castro HA, Hacon SS. Respiratory disease and climatic seasonality in children under 15 years old in a town in the Brazilian Amazon. J Pediatr (Rio J). 2008;84(6):543-549.

Artigo submetido em 16.05.08, aceito em 17.08.08.

doi:10.2223/JPED. 1846 


\section{Introdução}

As mudanças climáticas têm gerado preocupação crescente quanto aos potenciais efeitos à saúde humana, especialmente aqueles relacionados ao sistema respiratório. Alguns estudos têm mostrado a relação entre a variação sazonal e as proporções de atendimentos ambulatoriais por doenças respiratórias (DR), assim como a ocorrência de internações hospitalares ${ }^{1-5}$.

Vale ressaltar que a redução da umidade relativa do ar a valores abaixo de $30 \%$ é considerada de risco para a integridade das vias aéreas ${ }^{6}$, dificultando a homeostase interna do aparelho respiratório. Nos meses de chuva, em contraposição à problemática vivenciada nos meses de seca, a alta umidade relativa do ar, aliada ao maior tempo de permanência nos ambientes internos, ao menor arejamento e exposição ao sol dos espaços domiciliares, com conseqüente crescimento de mofo e fungos, são fatores que podem contribuir para o aumento das $D R$, especialmente as alérgicas ${ }^{6}$. Além disso, alguns vírus apresentam comportamento altamente sazonal, com maior freqüência no período frio em áreas de clima temperado e também no período de chuvas naquelas de clima tropical, como o da Amazônia legal. Esses vírus são freqüentemente causadores de DR em crianças, principalmente das vias aéreas superiores ${ }^{7,8}$. Entretanto, o conhecimento acerca do comportamento das DR em áreas de bioma amazônico e da possível influência dos ciclos típicos de seca e chuva sobre a saúde humana é escasso.

Em áreas urbanas industrializadas e/ou nas regiões de queima de biomassa, a baixa umidade nos períodos de estiagem soma-se à elevação dos níveis de poluição atmosférica. Os poluentes do ar iniciam um processo inflamatório no aparelho respiratório, alterando a permeabilidade das vias aéreas e possibilitando, assim, o acesso e a progressão de microorganismos patogênicos ${ }^{9}$. O material particulado presente na poluição do ar pode ainda interferir na depuração e inativação de bactérias que atingem o tecido pulmonar e contribuir para a ocorrência de doenças infecciosas ${ }^{10}$. Além disso, as crianças representam o grupo mais sensível aos efeitos dos poluentes atmosféricos.

Tangará da Serra (MT), área do presente estudo, encontra-se no trajeto de dispersão dos poluentes gerados tanto em países vizinhos quanto na área do arco do desmatamento $^{11}$. Possui ainda uma área crescente de cultivo de cana-de-açúcar, para abastecer três usinas de processamento na região. No período de seca, a queima de cana-deaçúcar é uma prática que resulta no aumento das concentrações de gases e partículas de aerossol na atmosfera da região. O município está inserido numa área de bioma amazônico e possui ciclos bem definidos de seca e chuva, que modificam os níveis de dispersão de poluentes atmosféricos ${ }^{12}$.

A seleção do município decorre desse importante cenário socioambiental, das perspectivas de crescimento do agronegócio para a região e da observação, entre 2000 e 2004, de um dos piores indicadores de morbimortalidade por DR em menores de 5 anos de idade do estado de Mato Grosso ${ }^{13}$. Sendo assim, a compreensão da variação dos atendimentos ambulatoriais relacionados à variação da umidade contribuirá para conhecimento do agravo à saúde mais freqüente na localidade.

Este estudo tem por objetivo analisar a sazonalidade climática dos atendimentos ambulatoriais por DR em menores de 15 anos.

\section{Métodos}

Estudo epidemiológico descritivo, realizado no município de Tangará da Serra (MT), por meio de boletins dos Registros de Ocorrências Ambulatoriais (ROA) das unidades básicas de saúde, do período de 2004 e 2005.

Tangará da Serra localiza-se a 240 km de Cuiabá, capital do estado de Mato Grosso. A estimativa populacional em 2005 foi de 70.258 habitantes, dos quais mais de $90 \%$ residem na área urbana. O Índice de Desenvolvimento Humano em 2000 era de 0,780 , um dos mais elevados do estado ${ }^{14}$. As principais atividades econômicas são a pecuária de corte e a agricultura de soja, algodão, café, arroz e cana-de-açúcar.

O município está ladeado pela Chapada dos Parecis e Serra Tapirapuã, com temperatura média de $25^{\circ} \mathrm{C}$ e umidade relativa do ar em torno de $80 \%$, podendo atingir $25 \%$ ou menos em período de seca extrema, em fins de julho/agosto ${ }^{15}$.

No período do estudo, o município encontrava-se habilitado em Gestão Plena da Atenção Básica Ampliada segundo a Norma Operacional da Assistência à Saúde, sendo responsável pela oferta de serviços apenas da atenção básica. O município implantou as três primeiras equipes de saúde da família no ano 2000, ampliando a rede para 10 unidades ao final de 2005. Atualmente, os atendimentos ambulatoriais da rede pública de saúde são realizados em oito postos convencionais, um centro de saúde, 10 unidades de saúde da família e uma unidade de emergência que realiza internações e cirurgias de baixa complexidade, denominada unidade mista.

Foram analisados todos os dados provenientes da atenção básica. Considerou-se de média complexidade os atendimentos realizados no centro de saúde, que é uma unidade de referência loco-regional para especialidades. Sendo assim, tais registros não foram incluídos no estudo. Foram excluídos, ainda, os registros de procedimentos, tais como curativos, vacinas e outros.

Não foi possível distinguir os atendimentos ambulatoriais referentes a consultas de rotina daqueles de emergência realizados na unidade mista de saúde, diante da ausência desse tipo de distinção anotada nos ROA.

\section{Análise dos dados}

Os atendimentos foram classificados por capítulos, de acordo com a $2^{a}$ versão da International Classification Primary Care (ICPC-2), e não de acordo com a Classificação Internacional de Doenças (CID-10), diante das limitações desta última na classificação dos atendimentos no âmbito da 
atenção primária ${ }^{16}$. As estimativas populacionais foram obtidas do Instituto Brasileiro de Geografia e Estatística (IBGE) ${ }^{14}$, e os dados de umidade relativa do ar, por meio do Instituto Nacional de Meteorologia (INMET), que possui uma estação automática em Tangará da Serra ${ }^{15}$.

Para conhecer a magnitude dos atendimentos por DR, dentre outros capítulos do ICPC-2, foi necessário construir um banco de dados contendo todos os atendimentos da atenção básica e de emergência ocorridos no município no ano de 2005. No entanto, para análise específica dos atendimentos por DR, foram agregados aos dados de DR do ano de 2005 aqueles de 2004. A construção de uma série de 2 anos de seguimento reduz a variabilidade dos registros, em conseqüência de especificidades tanto climáticas quanto dos serviços de saúde. Por se tratar de registros ambulatoriais, que em grande parte incluem sinais e sintomas sem diagnóstico definitivo, o diagnóstico não foi utilizado como variável em estudo. As variáveis de interesse foram: sexo, idade, localização anatômica do agravo, umidade relativa do ar e temperatura.

Optou-se por estudar os dados relativos aos menores de 15 anos, em razão de representarem aproximadamente $30 \%$ da população do município, bem como da maior vulnerabilidade biológica desse grupo etário, especialmente dos menores de 5 anos de idade.

Foram construídas taxas médias de atendimento ambulatorial por DR em menores de 15 anos segundo faixa etária e sexo para os anos de 2004 e 2005 . A idade foi categorizada em: < 1 ano, 1-4, 5-9 e 10-14 anos. Calculou-se, ainda, a razão seca/chuva do número de atendimentos de acordo com a localização anatômica da DR. Os períodos foram definidos como seca (maio a outubro) e chuva (novembro a abril). Para a classificação segundo a localização anatômica do agravo, foi utilizado o critério da CID-10 ${ }^{17}$, tendo a epiglote por limite de vias aéreas superiores/inferiores. As DR cujo sítio anatômico localizava-se acima da epiglote foram consideradas como doenças das vias aéreas superiores (DVAS), enquanto aquelas abaixo da epiglote (incluindo esta), foram consideradas como doenças das vias aéreas inferiores (DVAI). Foram categorizados como não especificados os sintomas relacionados ao trato respiratório (como tosse, por exemplo) sem definição diagnóstica.

Após autorização da Secretaria Municipal de Saúde de Tangará da Serra, os ROA foram fotocopiados, e todos os dados de interesse foram digitados. As diferenças de proporção foram testadas através do teste do qui-quadrado ao nível de significância de 5\%, com o programa Epi-Info 3.2.

\section{Considerações éticas}

Este estudo foi desenvolvido como produto do projeto de pesquisa intitulado "Avaliação dos efeitos da queima de biomassa na Amazônia legal à saúde humana", vinculado ao Instituto Milênio, que está inserido na rede Experimento de Grande Escala da Biosfera-Atmosfera na Amazônia (Large Scale Biosphere-Atmosphere Experiment in Amazonia -
LBA). Teve financiamento do Conselho Nacional de Desenvolvimento Científico e Tecnológico (CNPq) e da Fundação de Amparo à Pesquisa do Estado de Mato Grosso (FAPEMAT). $O$ estudo foi aprovado pelo Comitê de Ética em Pesquisa do Hospital Universitário Júlio Muller (HUJM), sob o no 290/CEPHUJM/06, em 24 de janeiro de 2007.

\section{Resultados}

Em 2005, foram registrados 36.344 atendimentos ambulatoriais pelo Sistema Único de Saúde (SUS) em Tangará da Serra, dos quais 6.895 em menores de 15 anos de idade (19\%). As doenças do aparelho respiratório representaram a maior proporção de atendimentos ambulatoriais no município $(15,8 \%)$, seguidas de condições relacionadas a gravidez, parto e planejamento familiar (13,2\%).

Enquanto, na população em geral, os atendimentos por DR em 2005 representaram menos de 1/5 do total de consultas médicas, os atendimentos em menores de 15 anos de idade responderam por $1 / 3$ dessas, com $32,7 \%$ do total de atendimentos (2.256/6.895).

Quando analisados os dados agregados de DR do período de 2004-2005, para a faixa etária de menores de 15 anos, foram obtidos 9.379 registros. A proporção de atendimentos por DR foi maior em crianças da faixa etária até 4 anos de idade (52\%), enquanto aquelas de 5 a 9 anos responderam por $28 \%$, e as de 10 a 14 anos, por cerca de $20 \%$ dos atendimentos.

Em relação à localização anatômica, $71 \%$ de todos os casos de DR foram decorrentes de DVAS (6.656), e 14,5\%, de DVAI (1.347); para 14,7\% dos casos, não foi possível especificar a classificação da localização anatômica (1.376).

Indivíduos do sexo masculino apresentaram taxa quase $50 \%$ maior de atendimentos ambulatoriais por DVAI que aqueles do sexo feminino (razão $=1,49$ ). Para as DVAS, essa razão foi de 1,03 (Tabela 1 ).

As taxas de atendimentos ambulatoriais por DR em menores de 15 anos variaram de $457,5 \%$ o nos menores de 1 ano de idade a $133,5 \%$ no grupo de 10 a 14 anos (Figura 1 ).

Quando comparados os períodos de seca e chuva, os atendimentos por DR foram em média $21 \%$ menos freqüentes no período seco (razão $=0,79$ ), valor estatisticamente significante (qui-quadrado $=122,2 ; p=0,000$ ). Ainda que haja uma proporção superior a $10 \%$ de DR não especificadas, a análise agregada, independente da localização anatômica, mostra maior proporção de atendimentos no período chuvoso. Os atendimentos por DVAS foram maiores no período chuvoso, entretanto para as DVAI não houve diferenças (Tabela 2).

A maior freqüência de atendimentos ocorreu nos meses de março e abril, final do período chuvoso, declinando a partir de então, para apresentar um pico no mês de agosto, com menor média de umidade relativa do ar. As menores temperaturas médias foram registradas nos meses de maio a julho, 
Tabela 1 - Razão de taxas para taxa média de atendimento ambulatorial em menores de 15 anos de idade segundo sexo e localização anatômica por 1.000 habitantes (Tangará da Serra, 2004-2005)*

\begin{tabular}{|c|c|c|c|c|c|}
\hline \multirow[b]{2}{*}{ Localização anatômica ${ }^{+}$} & \multicolumn{2}{|c|}{ Masculino } & \multicolumn{2}{|c|}{ Feminino } & \multirow[b]{2}{*}{ Razão de taxas } \\
\hline & $\mathbf{n}$ & Taxa & $\mathbf{n}$ & Taxa & \\
\hline DVAS & 3.302 & 157,38 & 3.151 & 152,02 & 1,03 \\
\hline DVAI & 792 & 37,34 & 528 & 25,02 & 1,49 \\
\hline Total & 4.094 & 225,97 & 3.679 & 207,61 & 1,08 \\
\hline
\end{tabular}

Fonte: Registros de Ocorrências Ambulatoriais, Secretaria Municipal de Saúde de Tangará da Serra, 2006. DVAI = doença das vias aéreas inferiores; DVAS = doença das vias aéreas superiores.

* A média da população masculina para o período foi de 10.497 crianças, e da feminina foi de 10.380 .

† Excluídas as doenças respiratórias sem especificação da localização anatômica.

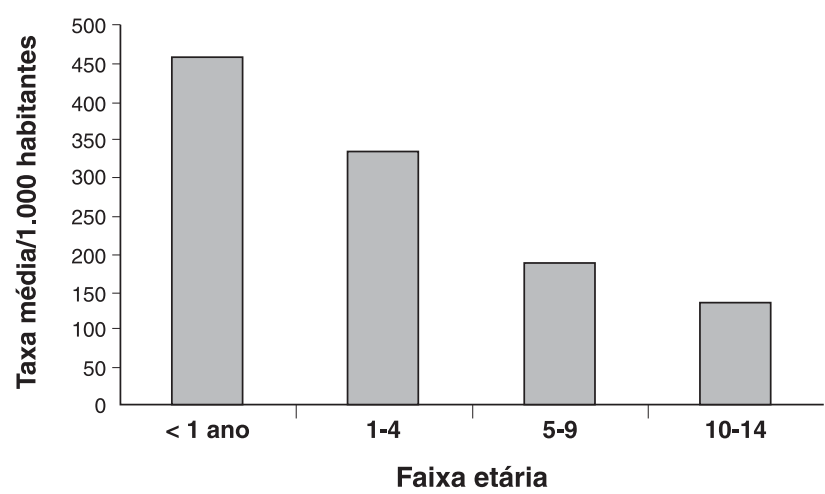

Fonte: Registros de Ocorrências Ambulatoriais, Secretaria Municipal de Saúde de Tangará da Serra, 2006.

Figura 1 - Taxa média de atendimentos ambulatoriais por DR segundo faixa etária (Tangará da Serra, 2004-2005)

quando também há uma redução da freqüência de atendimentos por DR (Figura 2).

Os atendimentos ambulatoriais realizados com maior freqüência no município de Tangará da Serra durante o período estudado decorreram de DR, especialmente em vias aéreas superiores e com maior magnitude em crianças abaixo de 5 anos de idade.

Estudo realizado no Brasil mostra não haver diferenças por sexo no uso de consultas médicas em crianças ${ }^{18}$. Entretanto, o sexo masculino tem sido descrito como fator de risco para DR em menores de 12 meses de idade ${ }^{19}$. Em Tangará da Serra, crianças do sexo masculino apresentaram maior proporção de consultas por DR, principalmente por DVAI, que configura os casos mais graves. Tal resultado corrobora os achados de Duarte \& Botelho, em estudo realizado em Cuiabá20.

As DVAS representaram a causa mais freqüentemente observada de atendimentos ambulatoriais em Tangará da Serra. Ainda que a proporção de DR não especificadas tenha sido superior a $10 \%$ e esse achado possa ou não modificar os resultados observados, deve-se considerar que no nível primário de atenção a codificação do diagnóstico nem sempre é precisa, uma vez que os problemas de saúde em atenção básica são indiferenciados e apresentam-se em diferentes estágios ${ }^{16}$. Possivelmente, o paciente busque o atendimento médico com um conjunto de sinais e sintomas ainda em fase inicial, cujo diagnóstico diferencial torna-se difícil. Entre o tempo demandado para exames laboratoriais e a consulta de retorno, pode ter havido remissão dos sintomas, com melhora clínica do paciente que não retorna aos serviços, impossibilitando a identificação do diagnóstico final.

A variação sazonal verificada neste estudo, com maior proporção de atendimentos ambulatoriais por DR em menores de 15 anos no período chuvoso, difere da variação observada por Rosa et al. ${ }^{5}$ nas internações hospitalares no município para a mesma faixa etária, cuja maior proporção ocorreu no período de seca.

A influência da sazonalidade climática nos atendimentos por DR, especialmente decorrente de DVAS no período das chuvas, sugere que os quadros mais benignos são responsáveis por maior proporção de atendimentos ambulatoriais, enquanto no período seco, o aumento de poluentes pode contribuir para a irritação das vias aéreas, facilitando o agravamento das DR. Estudo realizado por meio de registros de atendimentos de urgência por DR, em Cuiabá, mostrou maior freqüência dos mesmos durante o período chuvoso e, do mesmo modo, maior freqüência de internações durante a seca $^{1}$.

Em Mato Grosso, vive-se situação peculiar, em razão do ciclo de seca e chuva que marca as características ambientais. Durante o período seco, há importante aumento da poluição atmosférica, principalmente pelas queimadas de florestas, pastagens e reservas naturais de cerrado. Em Tangará da Serra, soma-se aos fatores descritos a queima da cana-de-açúcar e a poeira de ruas não pavimentadas.

Tanto a queda quanto a alta umidade relativa do ar nos períodos cíclicos de seca/chuva pode implicar dano ao apareIho respiratório dos indivíduos, principalmente para aqueles que têm algum acometimento prévio, tal qual um simples episódio de resfriado. 
Tabela 2 - Proporção média de atendimentos ambulatoriais segundo o período de seca e chuva (Tangará da Serra, 2004-2005)

\begin{tabular}{|c|c|c|c|c|c|c|c|}
\hline \multirow[b]{2}{*}{ Localização anatômica } & \multicolumn{2}{|c|}{ Seco } & \multicolumn{2}{|c|}{ Chuvoso } & \multirow{2}{*}{$\begin{array}{c}\text { Razão } \\
\mathbf{n}\end{array}$} & \multirow[b]{2}{*}{ Qui-quadrado } & \multirow[b]{2}{*}{$\mathbf{p}$} \\
\hline & $\mathbf{n}$ & $\%$ & $\mathbf{n}$ & $\%$ & & & \\
\hline DVAS & 2.820 & 42,43 & 3.836 & 57,57 & 0,73 & 148,57 & 0,000 \\
\hline DVAI & 704 & 52,26 & 643 & 47,74 & 1,09 & 2,76 & 0,09 \\
\hline Não especificado & 624 & 45,34 & 752 & 54,65 & 0,83 & 11,80 & 0,000 \\
\hline Total & 4.148 & 44,22 & 5.231 & 55,78 & 0,79 & 122,24 & 0,000 \\
\hline
\end{tabular}

Fonte: Registros de Ocorrências Ambulatoriais, Secretaria Municipal de Saúde de Tangará da Serra, 2006.

DVAI = doença das vias aéreas inferiores; DVAS = doença das vias aéreas superiores.
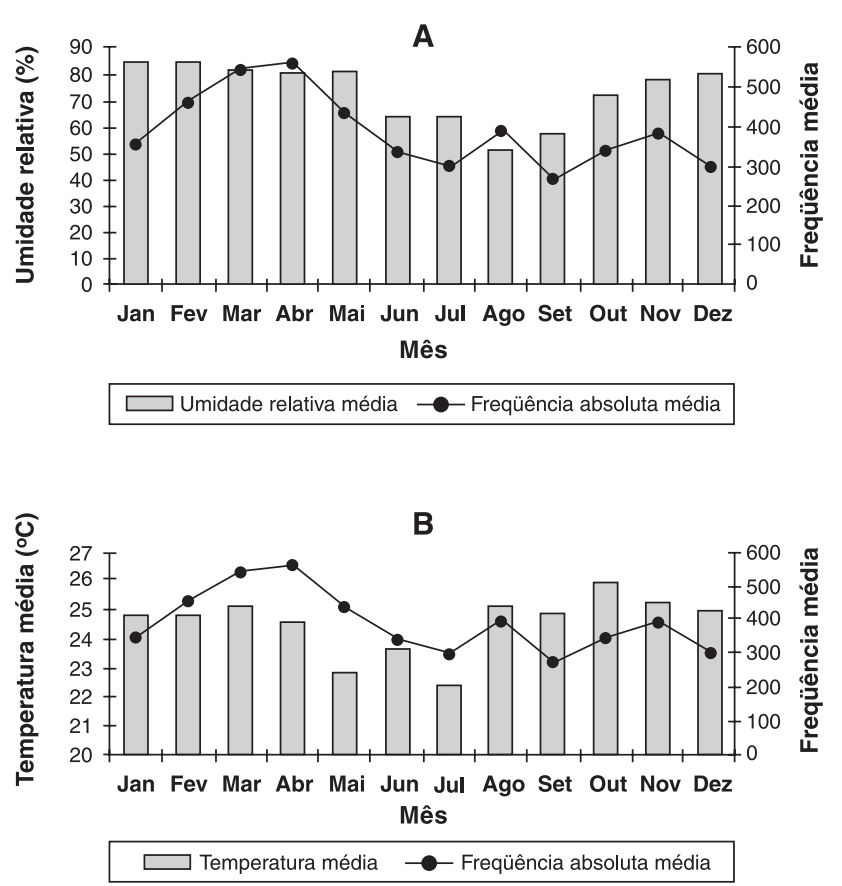

Fonte: Registros de Ocorrências Ambulatoriais, Secretaria Municipal de Saúde de Tangará da Serra, 2006. Instituto Nacional de Meteorologia, 2006.

Figura 2 - Média mensal do número de atendimentos ambulatoriais por $\mathrm{DR}$ em menores de 15 anos de idade, segundo o mês de ocorrência. A) Média mensal da umidade relativa (\%) do ar; B) temperatura média (Tangará da Serra, 2004-2005)

O paradoxo descrito pode ser evidenciado pelos picos de atendimento observados em março, período chuvoso, e em agosto, período seco. Entretanto, ainda que haja um pico de atendimentos em agosto, no mês de março o número de atendimentos é maior. Tais meses têm em comum o retorno das férias escolares, o que possivelmente influenciaria o volume observado de consultas médicas, em razão do maior contato das crianças com colegas portadores de microorganismos transportados dos espaços familiares ${ }^{21}$. Ainda que a variação da temperatura seja de apenas 5 a $6^{\circ} \mathrm{C}$ entre os meses mais quentes e os mais frios, a menor temperatura, aliada à menor umidade relativa do ar, parece contribuir para a redução na freqüência de atendimentos por DR no município no período estudado.

Observa-se também um fenômeno que pode ser denominado sazonalidade operacional, com queda na freqüência dos atendimentos da rede básica nos meses de dezembro a janeiro, assim como em fevereiro e julho, ainda que com menor relevância que dezembro e janeiro, provavelmente decorrente do período de férias dos profissionais de saúde, especialmente dos médicos.

Os ROA, a fonte de dados utilizada neste estudo, podem apresentar como limitação a qualidade dos registros, a falta de padronização e a validação de diagnósticos. No âmbito da atenção básica, podem ocorrer ainda um ou mais retornos em decorrência do mesmo problema de saúde. Esse fenômeno, muito mais operacional do que epidemiológico, não é passível de controle. Os ROA não apresentam registros fidedignos a respeito da continuidade do tratamento, nem tampouco um número no prontuário como elemento identificador.

Ainda que o estudo refira-se a dados secundários, cujos diagnósticos não foram validados, a ausência de vinculação do diagnóstico ao faturamento financeiro no nível básico de atenção reforça a importância dos resultados observados. Além disso, mesmo que houvesse alguma discordância em relação ao diagnóstico, parece pouco provável que tal discordância refira-se ao sistema anatômico acometido.

A população estudada representa apenas uma parcela dos residentes no município, não só por representar aqueles que são usuários do SUS, mas também porque os casos estudados referem-se àqueles que tiveram acesso à consulta médica ou que buscaram o atendimento. A busca por atendimento de saúde é influenciada por vários fatores, com algumas teorias explicativas para esse fenômeno, como a teoria comportamental proposta por Andersen \& Newman ${ }^{22}$, na qual determinantes individuais estão relacionados às características de acesso ao serviço, favorecendo ou limitando sua utilização.

Entretanto, os dados provenientes da atenção primária se referem a uma parcela mais ampla da população e apresentam maior possibilidade de identificação dos casos de doenças que possam estar relacionadas a fatores de risco, como 
poluição do ar atmosférico, por exemplo, em fases muito iniciais da doença. Além disso, resultados da Pesquisa Nacional por Amostra de Domicílios mostram que, em média, 24,5\% da população do país possui algum plano privado de saúde ${ }^{23}$. Possivelmente, tanto as consultas ambulatoriais quanto as hospitalizações pelo SUS representem a maioria dos atendimentos no país, como observado por da Silva et al. ${ }^{24}$, ainda que haja variações regionais na dependência do sistema público de saúde.

Considera-se necessário ampliar a compreensão acerca do comportamento das DR na região da Amazônia brasileira, bem como estabelecer estratégias para a validação desses diagnósticos e para a identificação dos fatores de risco, que podem ou não ser diferentes daqueles relatados na literatura. Ainda que fuja ao escopo deste estudo, todas essas questões merecem futuras avaliações e fazem parte da agenda do grupo de pesquisa.

Frente ao cenário ambiental descrito e às condições de saúde infantil observadas no município, é relevante considerá-lo como área de atenção ambiental atmosférica de interesse para a saúde e estabelecer uma rede integrada de atenção saúde-ambiente local.

Os ROA poderão ser utilizados como fonte de dados para a construção de indicador sentinela de fatores de risco, subsidiando tanto as ações da vigilância de saúde-ambiente quanto o processo decisório. Esses indicadores deverão ser estabelecidos a partir de discussões com os atores sociais, para a seleção daqueles mais sensíveis na identificação de casos e de alterações na tendência de distribuição dos mesmos. A redução da vulnerabilidade dos sistemas socioambientais requer maior capacidade de organização dos serviços e melhor uso dos recursos disponíveis ${ }^{25}$, bem como compreensão da dinâmica das relações de produção e consumo na comunidade, com enfoque multidisciplinar ${ }^{26}$.

Sugere-se ainda a instituição de medidas para promoção da saúde, vigilância dos fatores de risco, identificação precoce de casos, manejo padronizado e acompanhamento adequados a partir de estratégias adotadas nacional e internacionalmente, como, por exemplo, a Atenção Integrada às Doenças Prevalentes na Infância.

Conclui-se que os atendimentos ambulatoriais por DR, especialmente aqueles por DVAS, comumente de menor gravidade, estão relacionados ao período da chuva no município.

\section{Referências}

1. Botelho C, Correia AL, da Silva AM, Macedo AG, Silva CO. Fatores ambientais e hospitalizações em crianças menores de cinco anos com infecção respiratória aguda. Cad Saude Publica. 2003; 19:1771-80.

2. Façanha MC, Pinheiro AC. Doenças respiratórias agudas em serviços de saúde entre 1996 e 2001, Fortaleza, CE. Rev Saude Publica. 2004;38:346-50.
3. Valença LM, Restivo PC, Nunes MS. Variação sazonal nos atendimentos de emergência por asma em Gama, Distrito Federal. J Bras Pneumol. 2006;32:284-9.

4. González DA, Victora CG, Gonçalves H. Efeitos das condições climáticas no trimestre de nascimento sobre asma e pneumonia na infância e na vida adulto em uma coorte no sul do Brasil. Cad Saude Publica. 2008;24:1089-102.

5. Rosa AM, Ignotti E, Hacon SS, Castro HA. Análise das internações por doenças respiratórias em Tangará da Serra Amazônia Brasileira. J Bras Pneumol. 2008;34:575-82.

6. Mold. Una breve guía para el moho la humedad y su hogar. Agency. [site na internet]. Washington, DC, United States Environmental Protection; 2008. http://www.epa.gov/mold/ moldresources.html. Acesso: 18/04/2008.

7. Thomazelli LM, Vieira $S$, Leal AL, Sousa TS, Oliveira DB, Golono MA, et al. Surveillance of eight respiratory viruses in clinical samples of pediatric patients in southeast Brazil. J Pediatr (Rio J). 2007; 83:422-8.

8. Loscertales MP, Roca A, Ventura PJ, Abacassamo F, Dos Santos F, Sitaube M, et al. Epidemiology and clinical presentation of respiratory syncytial virus infection in a rural area of southern Mozambique. Pediatr Infect Dis J. 2002;21:148-55.

9. Correia-Deur JE. Contaminación del aire exterior y enfermedades alérgicas de la vía aérea. Cienc Trab. 2007; 9:23-9.

10. Nel A. Atmosphere. Air pollution-related illness: effects of particles. Science. 2005;308:804-6.

11. Silva Dias MA, Cohen JC, Gandú AW. Interações entre nuvens, chuvas e a biosfera na Amazônia. Acta Amaz. 2005;35:215-22.

12. Monitoramento de emissões de poluentes atmosféricos e previsão da qualidade do ar. [site na internet]. Cachoeira Paulista, SP: Centro de Previsão de Tempo e Estudos Climáticos/Instituto Nacional de Meteorologia - CPTEC/INPE; 2007. http:// www.cptec.inpe.br. Acesso: 20/03/2007.

13. Ignotti E, Hacon SS, Silva AM, Junger WL, Castro HA. Efeitos das queimadas na Amazônia: método de seleção dos municípios segundo indicadores de saúde. Rev Bras Epidemiol. 2007; $10: 453-64$

14. Instituto Brasileiro de Geografia e Estatística - IBGE. [site na internet]. Brasília, DF; 2007. www.ibge.gov.br. Acesso: 20/03/ 2007.

15. Estação meteorológica de observação de superfície. [banco de dados na internet]. Brasília (DF): Instituto Nacional de Meteorologia - INMET; 2007. http://www.inmet.gov.br/sonabra/ dbRegSonabra.php?codEst $=$ A902\&dtaini $=16 / 08 / 2008 \&$ dtafim $=16 / 08 / 2008 \&$ Submit $=$ Pesquisar. Acesso: $20 / 05 / 2007$.

16. WONCA - Comissão de Classificações da Organização de Ordens Nacionais, Academias e Associações Acadêmicas de Clínicos Gerais/Médicos de Família. Classificação Internacional dos Cuidados Primários. 2a. ed. Oxford: Oxford University Press; 1999. http://www.grunenthal.pt/cw/pt_PT/pdf/cw_pt_pt_ icpc2.pdf. Acesso: 26/02/2007.

17. Organização Mundial de Saúde. Centro colaborador da OMS para a Classificação de Doenças em Português (trad.). Classificação internacional de doenças - $10^{a}$ revisão (CID-10). $5^{a}$ Ed. São Paulo: USP; 1997.

18. Ribeiro MM. Utilização de serviços de saúde no Brasil: uma investigação do padrão etário por sexo e cobertura por plano de saúde [dissertação]. Belo Horizonte (MG): Universidade Federal de Minas Gerais; 2005. 
19. Macedo SE, Menezes AM, Albernaz E, Post P, Knorst M. Fatores de risco para internação por doença respiratória aguda em crianças até um ano de idade. Rev Saude Publica. 2007; 41:351-8.

20. Duarte DMG, Botelho C. Perfil clínico de crianças menores de cinco anos com infecção respiratória aguda. J Pediatr (Rio J). $2000 ; 76: 207-12$.

21. Nesti MM, Goldbaum M. Infectious diseases and daycare and preschool education. J Pediatr (Rio J). 2007;83:299-312. Epub 2007 Jul 13.

22. Andersen R, Newman JF. Societal and individual determinants of medical care utilization in the United States. Milbank Mem Fund Q Health Soc. 1973;51:95-124.

23. Pesquisa Nacional por Amostra de Domicílio/PNAD. Acesso e utilização de serviços de saúde. Brasília: Instituto Brasileiro de Geografia e Estatística - IBGE; 2003. www.ibge.gov.br. Acesso: 20/03/2007.
24. da Silva AA, Gomes UA, Tonial SR, Silva RA. Fatores associados à realização de consultas médicas de crianças menores de 5 anos. Rev Bras Epidemiol. 1999;2:60-72.

25. Sabroza PC. Estudos epidemiológicos na perspectiva do aumento da vulnerabilidade dos sistemas sócio-ambientais brasileiros. Epidemiol Serv Saude. 2007;16:229-32.

26. Kligerman DC, Vilela H, Cardoso TA, Cohen SC, Sousa D, La Rovere E. Sistemas de indicadores de saúde e ambiente em instituições de saúde. Cien Saude Colet. 2007;12:199-211.

Correspondência:

Antonia Maria Rosa

Av. Getúlio Vargas, 2335, Jardim do Trevo

CEP 78200-000 - Cáceres, MT

Tel.: (65) 9601.3465, (65) 3221.0504

E-mail: antonia-mr@unemat.br, antonia-mr@hotmail.com 